\title{
Age-related Histological Changes in the Renal Telocytes in Male Albino Rats
}

\section{Abeer Madkour Mahmouda, Dorreia Abdallah Mohamed Zaghloul ${ }^{b}$, Fatma Y. Meligy ${ }^{\mathrm{c}}$, Maha AA} Fahmy .

${ }^{a}$ Human Anatomy \& Embryology Department, Faculty of Medicine, South Valley University, Qena, Egypt.

${ }^{\mathrm{b}}$ Human Anatomy and Embryology Department, Faculty of Medicine, Assuit University, Assiut, Egypt.

${ }^{c}$ Histology and Cell Biology Department, Faculty of Medicine, Assuit University, Assiut, Egypt.

\section{Abstract}

Background:Many researches on telocytes (TCs) have proved its existence, structure and specific immune markers expression in many normal organs and in different species. Multiple physiologic functions have been suggested for these cells.

Objectives: This study is designed to evaluate age-related changes ofTCs in different age groups in the renal tissue.

Materials and Methods: 18 male Sprague- Dawley rats were used in this study and divided equally into 3 groups (each consists of 6 rats); Group I (rats aged 3 weeks), Group II (rats aged 3 months, adult) and Group III (rats aged one year). All rats of group I, II and III were sacrificed and each group was divided into two subgroups. The first subgroup was processed for light microscopic and immunohistochemical staining. The second subgroup was processed for electron microscopy. Morphometric and statistical studies were conducted.

Results: TCs with their characteristic long and thin cytoplasmic projections (telopodes, Tps) are detected in each of H\&E stained and CD34 immunostained sections. In group III, there is a significant reduction of the TCs count. TEM examination of the ultrathin sections revealed shrinkage of TCs with disintegration and fragmentation of the Tps with a significant reduction of the number of Tps arising from each TC in group III. In all groups of the study, homocellular and heterocellular junctions are observed.

Conclusion: On comparison of the age groups in the study, it is proved that TCs decrease in number with degenerative changes observed with aging.

Keywords: Telocytes, Telopodes, Renal Interstitium, CD34.

\section{Introduction}

A new stromal interstitial cell type with peculiar ultrastructural features is called telocyte (TC) has been described in various organs of different species as cell with very long cellular extensions called telopodes with alternation of thin segments(podomers) and dilated portions (podoms) (Zhang et al., 2016; Cretoiu et al., 2017).

Increasing evidence indicates that TCs can construct a complex three-dimensional network within the stromal compartment and release a variety of extracellular vesicles to regulate the functions of surrounding cells (Cismasiu and Popescu, 2015; Cretoiu et al., 2016; Marini et al., 2017).

TCs are suggested to play roles in tissue structural support, homeostasis maintenance, intercellular signaling, cell differentiation, immune surveillance, stem/progenitor cell guiding and nursing, and to participate in different tissue 
pathologies. Especially, TCs are believed important in tissue regeneration, making them particularly attractive in the field of regenerative medicine (Wang et al., 2020; Liao et al., 2021).

The kidney is a very specialized organ; histological structure of the kidney shows more than thirty different cell types. Each cell type has its own proliferation rate and regenerative capacity. Epithelial cells lining inner layer of the Bowman's capsule "podocytes", have no proliferative capacity. If these cells are lost, they cannot be replaced. Cells of the proximal tubules have a low physiological turnover capacity. Upon pathological damage, these tubular cells respond with diffuse proliferation (Bussolati et al., 2009; Song et al., 2012).

It has been reported that immunohistochemical staining and light microscopy techniques can be used to quantify the morphology of telocytes in various systems of human and different animals (Xu et al., 2019). CD34immunostaining was documented to be the most convenient marker used for detection, differentiation of TCs from other surrounding cells and counting them in the intertubular interstitial spaces in renal cortex, where CD34 was expressed in the cytomembrane(Faussone-Pellegrini and Popescu, 2011; Gandahi et al., 2020).

\section{Materials and Methods}

\section{1-Animals}

The study was conducted on 3 different ages of male Sprague- Dawley rats with the age of 3 weeks, 3 months (adult weighing 170- $250 \mathrm{gm}$ ) and one year (late adult). Rats in this study were obtained from animal house, Faculty of Medicine, Assiut University. Rats were maintained on a 12 hours light/dark cycle at $21 \pm 1^{\circ} \mathrm{C}$ and $50 \pm 10 \%$ humidity. The rats were kept under hygienic conditions, housed in metal cages, and bedded with wood shavings, fed libitum and all had a free access to water.

18 male Sprague- Dawley rats were used in this study and divided equally into 3 groups:

Group I: consisted of 6 rats at age of 3 weeks.
Group II: consisted of 6 rats at age of 3 months (adult)

Group III: consisted of 6 rats at age of one year.

All rats of group I, II and III were anesthetized, sacrificed and each group was divided into two subgroups. The first subgroup was processed for light microscopic study and immunohistochemical staining where animals were perfused with buffered formalin and specimens from the kidney were fixed in $10 \%$ buffered formalin for 24 hours. The second subgroup was processed for electron microscopy where animals were subjected to an intracardial perfusion of $2.5 \%$ glutaraldehyde then kidneys were trimmed and fixed in $2.5 \%$ glutraldehyde.

\section{2- Histological techniques:}

\section{a-Histological techniques for light microscopic study:}

The histological sections were stained by the following stains (Bancroft and Layton, 2013):

1-Haematoxyline and Eosin stain (H\&E)

2- Immunohistochemical staining for detection of CD34 positive cells.

Photographs of the examined slides were carried out with a Leica microscope fitted with a digital camera.

b- Histological technique for semi-thin and ultrathin section (Ayache et al., 2010):

a) Semithin sectioning at thickness of 1um: Specimens were trimmed with a razor blade and stained with $2 \%$ aqueous toluidine blue then dried on a hot plate at $40 \mathrm{c}$.

b) Ultrathin sectioning at thickness of $50 \mathrm{~nm}$ were recommended on coper grids examined by a transmission electron microscope (TEM) and photographed at the electron microscope unit of Assiut university.

\section{3- Morphometric measurements:}

morphometric analysis of CD34 immunostained sections was done counting the TCs using an objective lens (X40) in four nonoverlapping fields in ten randomly chosen sections from three different animals from each group using computer-assisted image analysis software (Leica 
microsystems CMS GmbH, Wetzlar, Germany) in the department of human anatomy, faculty of medicine, South Vally University.

Morphometric measurements of other parameters as the TCs cell body length (um),TCs nucleus diameter (um), length of Tps (um) and number of Tps emerging fromeach TC, are obtained by applying the imageJ software on the transmission electron micrographs. A set scale was used to insert the value of the scale bar tobe able to determine the surface area and measure the mentioned parameters.

\section{4- Statistical analysis:}

All values of themorphometric analysis of TCs in CD34 immunostained sections and TEM micrographsare given as mean $\pm \mathrm{SD}$ in each group.The data will be analyzed using SPSS version 20.0 statistical software. Different unpaired $t$-test was used to compare variables, $\mathrm{P}$ value $<0.05$ was considered statistically significant.

\section{Results:}

\section{1- Haematoxyline and Eosin stain (H\&E)}

Examination of $H \& E$ stained sections of renal tissue particularly renal cortex of group Iand group II revealed normal renal tubules (proximal and distal convoluted tubules), glomeruli and blood vessels; in addition to telocytes (TCs), presenting with their characteristic long and thin cytoplasmic projections (telopodes, Tps), and cylindrical cell bodies were detected. They were found in-between the renal tubules and in close proximity to blood vessels (Fig.1\&2). Tpswere noticed to extend forming networks through which TCs showed contact with other TCs and surrounding cells. In group III, in addition to the renal tubular changes related to the renal aging (in the form of presence of vacuolation in the cells of renal tubules, degenerated cells, and congestion of blood vessels), the examination of $H \& E$ stained sections of the renal cortical tissue reveals TCs with their characteristic long and thin Tps and cylindrical cell bodies. They were found in-between the renal tubules and in close proximity to blood vessels but less frequent than in groups I \& II (Fig.3).

\section{2- Immunohistochemical staining:}

Light microscopic examination of CD34 immunohistochemical stained sections in group I and II revealed CD34-positive TCs with a high expression in cell bodies and Tps. TCs and their Tps often were chained in networks surrounding the microvessels and in-between the renal tubules (Figs $4 \& 5)$. In group III, examination of CD34immunostained sections showed weak positive CD34 - immunoreactive TCs with their Tps inbetween the renal tubules (Fig.6). Morphometric measurements and analysis of TCs have been done through counting TCs in group III immunostained sections, where mean $\pm \mathrm{SD}$ of the number of TCs was $1.83 \pm 0.81$. There was a significant decrease (P-value $<0.0001)$ in number of TCs in group III in comparison with those in both of group I and group II.

Morphometric study and analysis of TCs were accomplished through counting TCs in immunostained sections (table $1 \&$ chart 1), where the number of TCs in group I (mean \pm SD) was 5.05 \pm 1.20 . Mean \pm SD of the number of TCs in group II was $3.0 \pm 0.93$. There was significant decrease (P-value $<0.0001)$ in number of TCs in group II in comparison with those in group I. In group III, mean \pm SD of the number of TCs was $1.83 \pm 0.81$. There was significant decrease (P-value $<0.0001)$ in number of TCs in group III in comparison with those in both of group I and group II.

\section{3- Semithin sections:}

Examination of semithin sections of each of group I and II stained with toluidine blue showed TCs with their characteristic Tps in-between the renal tubules and in close proximity to blood vessels and glomeruli (Fig.7\&8).In group III, TCs were identified, but they were few and far between (Fig.9).

\section{4- Transmission electron microscopic examination:}

In group I and group II, TCs with their long Tps could be clearly seen in the renal cortex in between the renal tubules and near the blood vessels.TCs display an indented oval shaped nucleus showing patches of heterochromatin 
particularly near the nuclear membrane in addition to euchromatin. The nucleus was relatively large surrounded by a thin cytoplasmic layer containing few mitochondria, few endoplasmic reticulum, filaments and a small Golgi apparatus (Fig.10,11\&12). In group III, through the TEM examination, TCs with their Tpswere rare as they were found with difficulty in the renal cortical tissue. TCs displayed an oval shaped nucleus showing euochromatin distributed throughout the nucleus in addition to disorganized heterochromatin near the nuclear membrane. The nucleus was relatively large surrounded by a thin cytoplasmic layer containing few mitochondria, few endoplasmic reticulum, filaments and a small Golgi apparatus. Intracellular and extracellular vesicles in addition to caveolae could be clearly seen (Fig.13). Comprehensive EM examination of specimens of group III revealed the presence of disintegrated TCs with fragmentation of their Tps.

Morphometric analysis of TCs and their Tpswas done using the imagej software on the TEM photomicrographs (table1\&charts 2-5).

Telopodesin groups I and II were detected as discontinuous segments emerging from the cellular body with alternation of podoms and podomers. Podoms constituted the dilated portion, which contain mitochondria, endoplasmic reticulum, secretory vesicles, caveolae and filaments. Podomerswere thin processes between the podoms. In group III, frequently Tpswere found to be disintegrated and fragmented. Secretory vesicles and caveolae were detected on ultrastructural examination of group III Tps.

In all groups of the study, homocellular and heterocellular junctions were noticed between TCs cell bodies with their Tps and the neighboring TCs, the cells of renal tubules and vascular endothelial cells (Fig.11,12\&13).

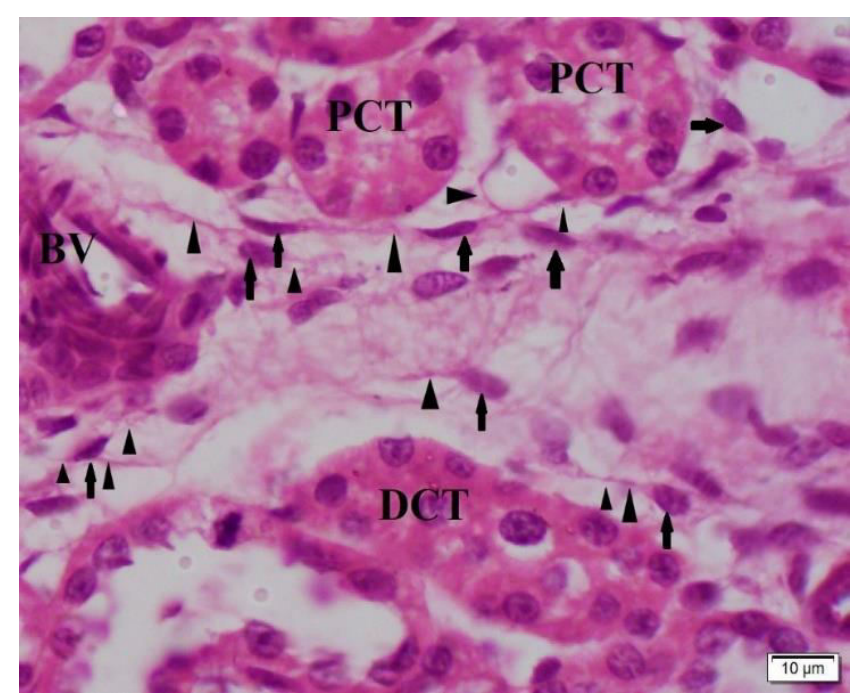

Fig1: A photomicrograph of a section from the kidney in the group I (3 week-aged), showing telocytes (black arrows) with their telopodes (black arrowheads) located near renal tubules and in the proximity of blood vessel (BV) (proximal convoluted tubule, PCT; distal convoluted tubule, DCT) (H\&E stain with magnification $\times 1000$ ).

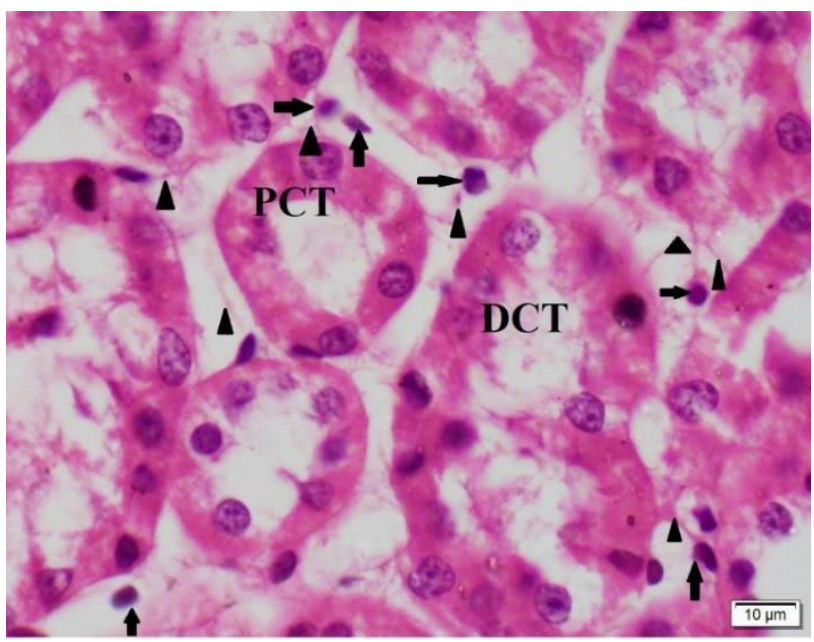

Fig 2: A photomicrograph of a section from the kidney in the group II (adult), showing telocytes (black arrows) with their telopodes (black arrowheads) located in between renal tubules. (Proximal convoluted tubule, PCT; distal convoluted tubule, DCT) (H\&E stain with magnification x1000). 


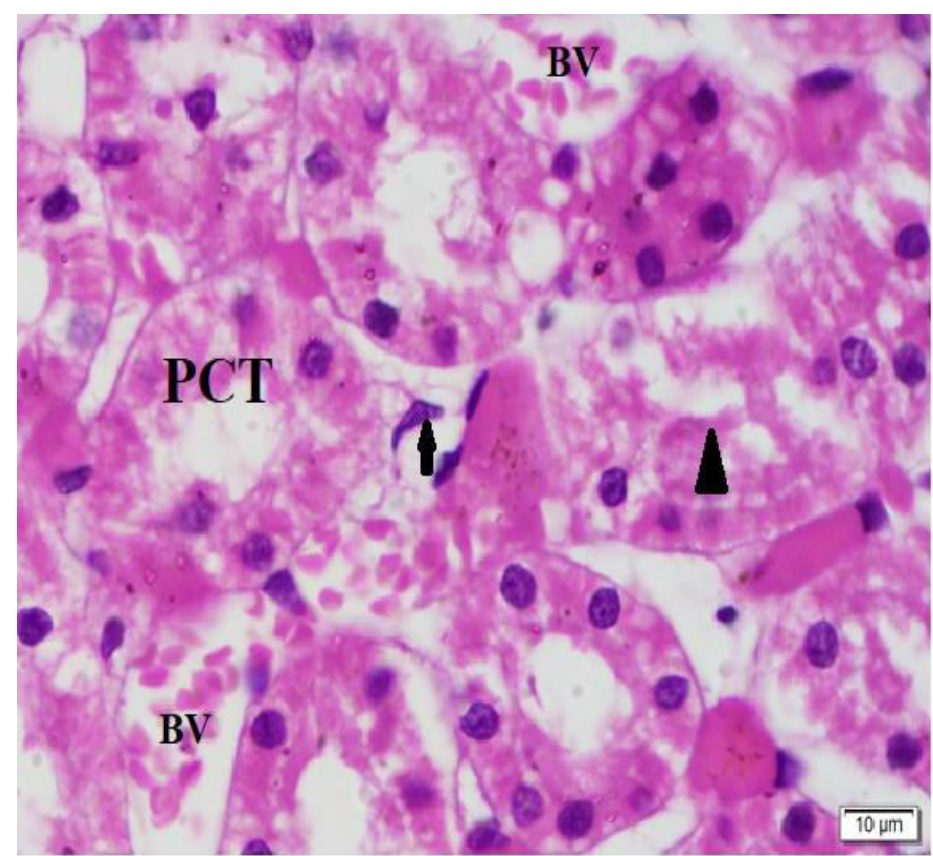

Fig 3: A photomicrograph of a section from the kidney in the group III (1 year-aged), showing a degenerated renal tubule (arrowhead). Congestion of the blood vessels (BV) is present. A telocyte is observed (black arrow) with Tps extending from it. (Proximal convoluted tubule, PCT) (H\&E stain with magnification $\mathrm{x} 1000$ ).

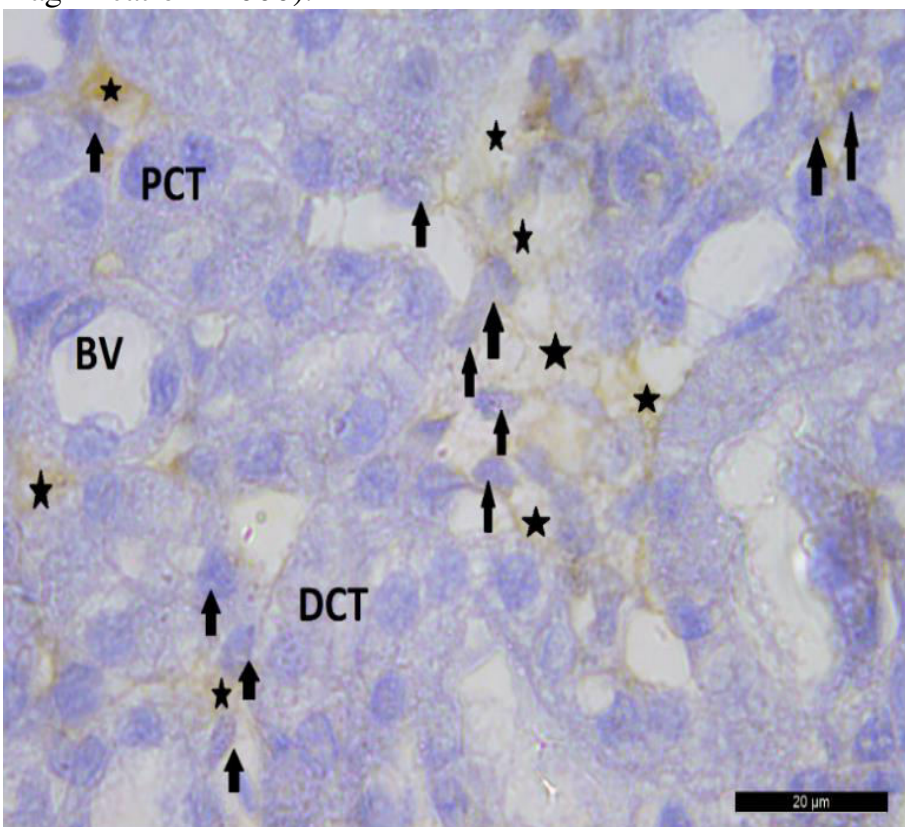

Fig 4: A photomicrograph of a CD34-immunostained section from the kidney in the group I (3 week-aged), TCs showed a positive reaction for CD34 immunostaining (black arrows) with their telopodes (Asterisks) forming networks in between the renal tubules and in the proximity of blood vessel (BV). (Proximal convoluted tubule, PCT; distal convoluted tubule, DCT; glomerulus, G) (magnification x1000).

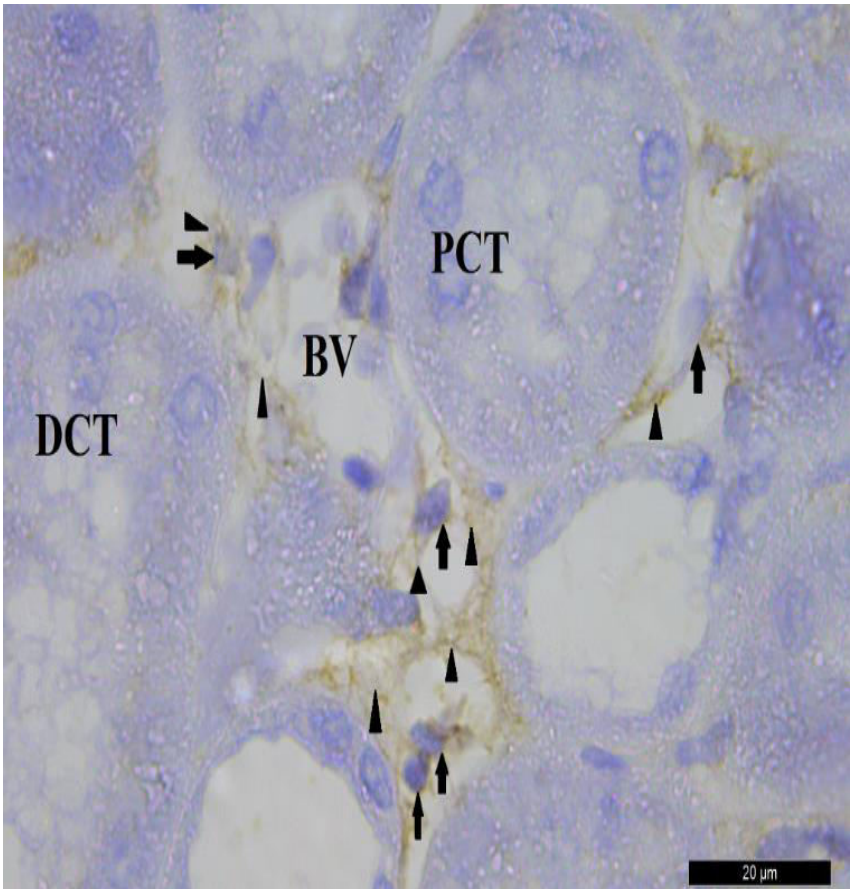

Fig 5: A photomicrograph of a CD34-immunostained section from the kidney in the in group II, TCs showed a positive reaction for CD34 immunostaining (black arrows) with their telopodes (arrowheads) located in between the renal tubules and in the proximity of blood vessel (BV). (Proximal convoluted tubule, PCT; distal convoluted tubule, DCT; glomerulus, G) (magnification x1000).

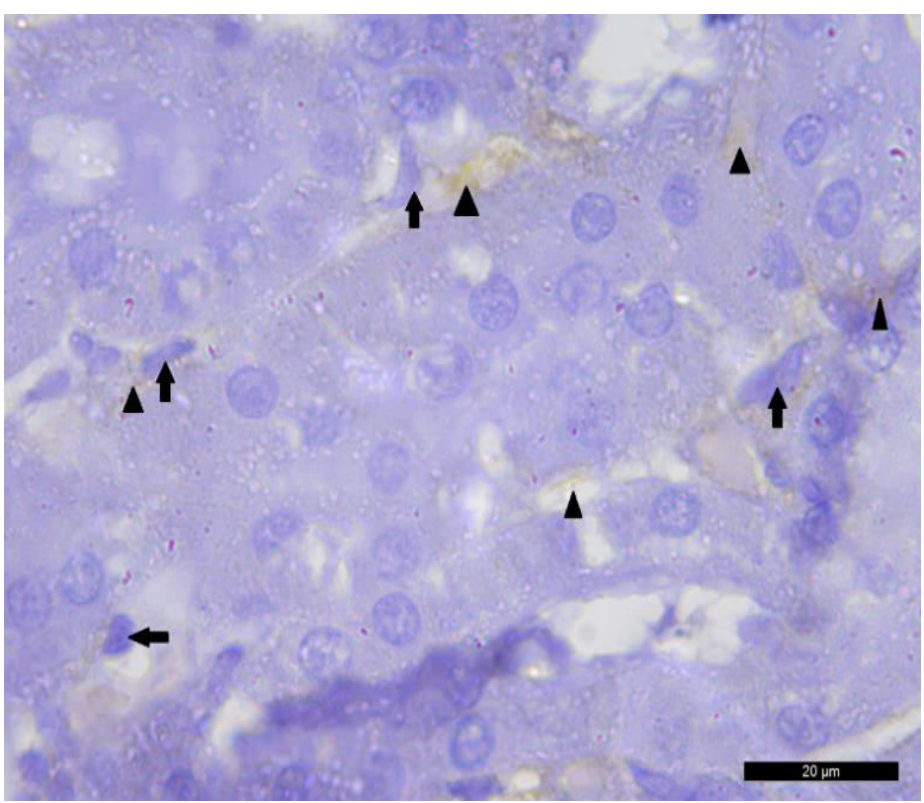

Fig 6: A photomicrograph of a CD34-immunostained section from the kidney in group III. TCs show a weak positive reaction for CD34 immunostaining (black arrows) located in between the renal tubules, with their telopodes (arrowheads) forming networks. (magnificationx1000). 


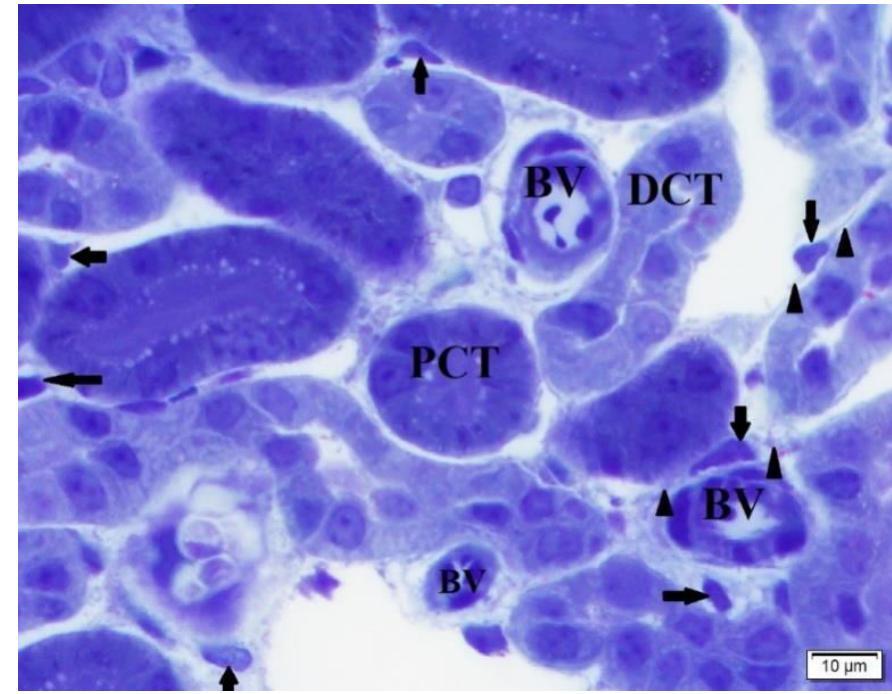

Fig.7: A photomicrograph of a semithin section from the kidney in the group I (3 week-aged), showing telocytes (black arrows) with their telopodes (black arrowheads) located in between the renal tubules and in the proximity of blood vessel (BV). (proximal convoluted tubule, PCT; distal convoluted tubule, DCT) (toluidine blue stain with magnification x1000).

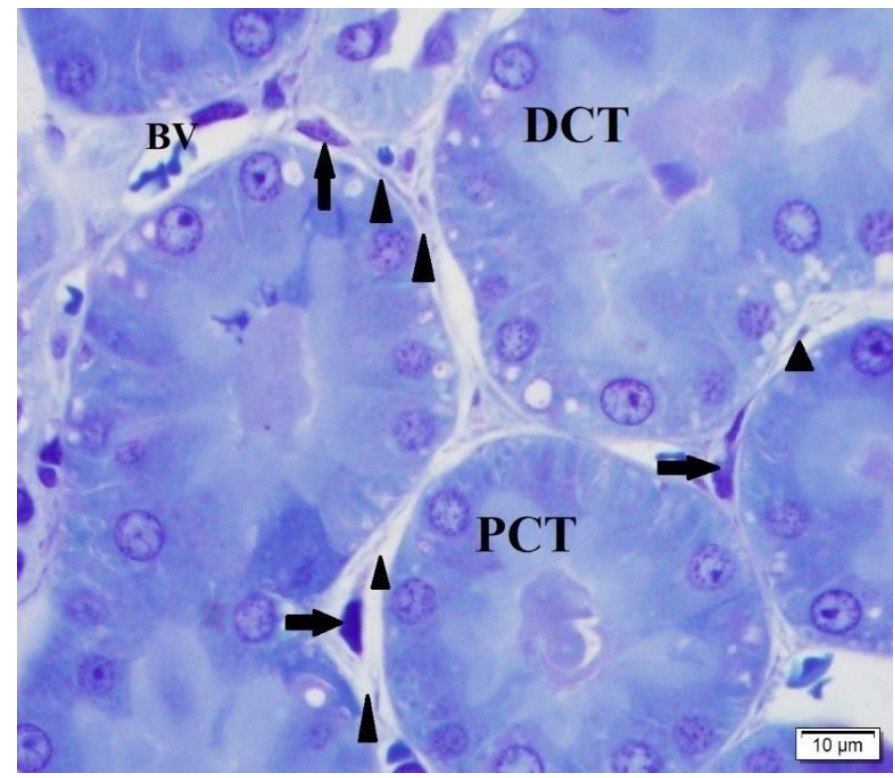

Fig.8: A photomicrograph of a semithin section from the kidneyin group II, showing telocytes (black arrows) with their telopodes (black arrowheads) located in between the renal tubules and in the proximity of blood vessel (BV). (Proximal convoluted tubule, PCT; distal convoluted tubule, DCT) (toluidine blue stain with magnification $\mathrm{x} 1000$ ).

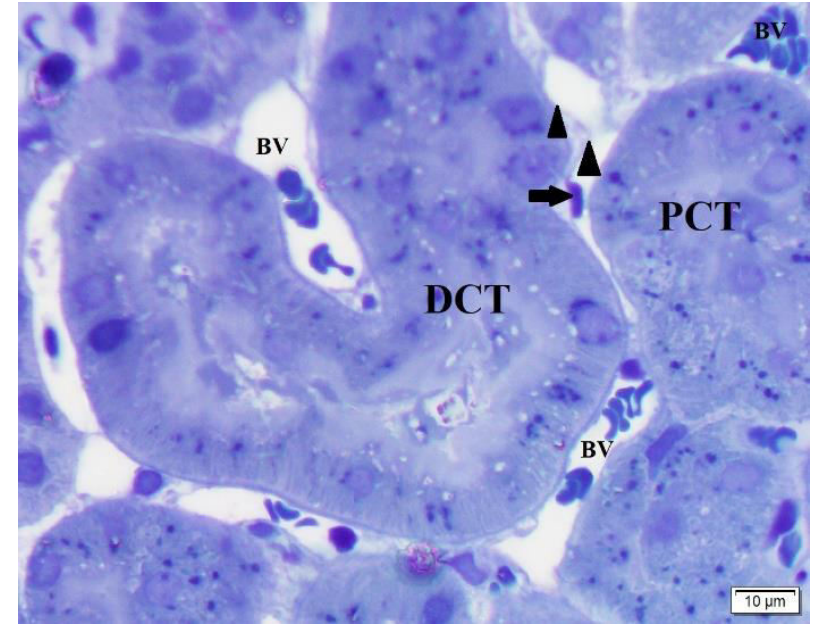

Fig. 9: A photomicrograph of a semithin section from the kidneyin group III, showing the renal tubules with decreased density of staining and extrusion of some nuclei into the lumina of the tubules. Pigment deposition is observed in the tubular epithelium (the deposited pigments are considered to be lipofuscin). Telocyte (black arrows) with their telopodes (black arrowhead) located in between the renal tubules and in the proximity of blood vessel (BV). Congestion of the blood vessels is present. (Proximal convoluted tubule, PCT; Distal convoluted tubule, DCT) (toluidine blue stain with magnification $\mathrm{x} 1000$ ).

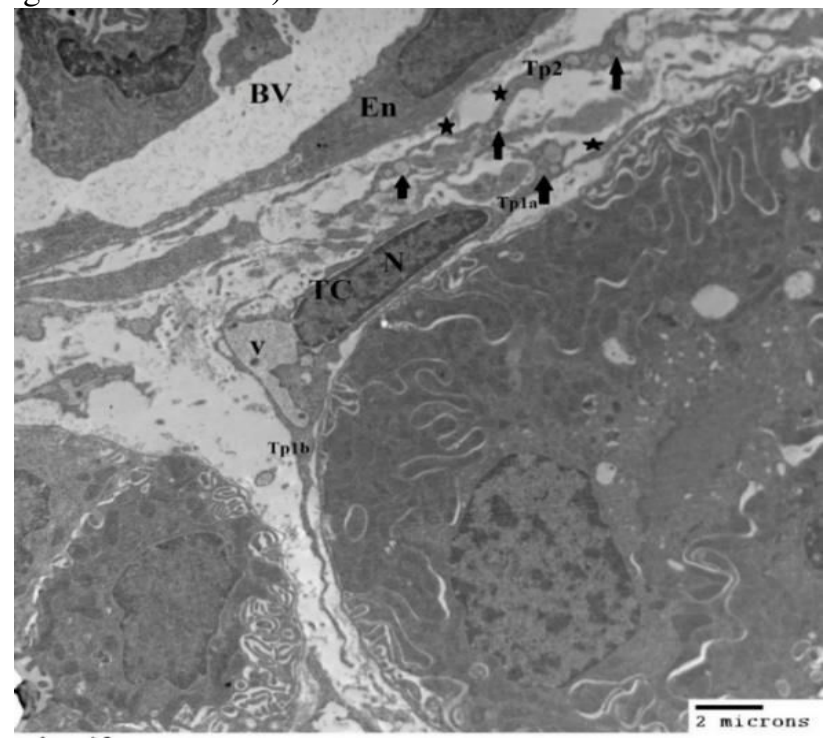

Fig.10: TEM photomicrograph of ultrathin section from the kidney in the group I (3 week-aged). Telocyte (TC) is present in between the renal tubules and blood vessels. TC display a relatively large indented oval nucleus $(\mathrm{N})$ showing patches of heterochromatin particularly near the nuclear membrane in addition to euchromatin. The nucleus is surrounded by a thin cytoplasmic layer. A large intracytoplasmic vesicle (v) is present. Telopodes (Tp1a and Tp1b) arise from the TC. Telopode (Tp2) of another TC is present. Tps show alternating podoms (arrows) containing mitochondria and vesicles, and 
podomeres (Asterisks). (Blood vessel, BV; endothelial cell, En; scale bar: 2 um, x3600).

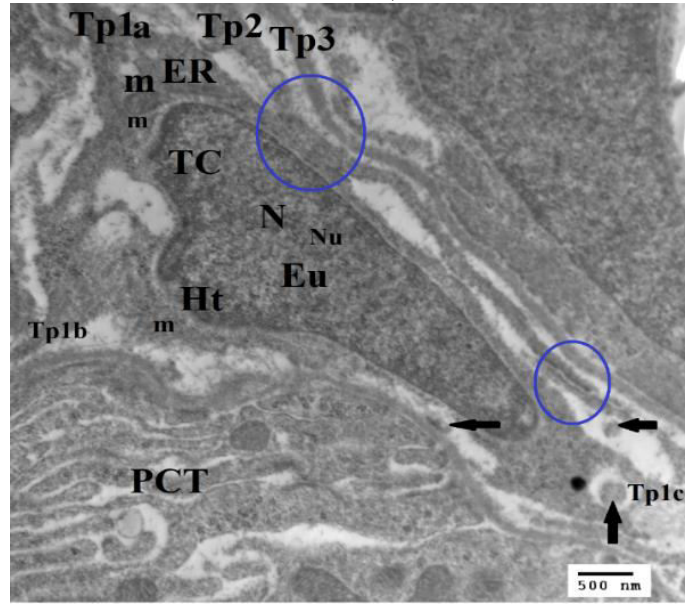

Fig.11: TEM photomicrograph of ultrathin section from the kidney in the group I (3 week-aged). Telocyte (TC) display a relatively larger indented oval nucleus $(\mathrm{N})$ showing patches of heterochromatin $(\mathrm{Ht})$ particularly near the nuclear membrane in addition to euchromatin $(\mathrm{Eu})$, nucleolus $(\mathrm{Nu})$ is apparant. The nucleus is surrounded by a thin cytoplasmic layer containing few mitochondria $(\mathrm{m})$, few endoplasmic reticulum (ER). Telopodes (Tp1a, Tp1b and Tp1c) arise from the TC. Homocellular junction (blue circles) appears where the TPs of the TC form junctions with Tps (Tp2 and Tp3) of other adjacent TCs. Intra- and extra-cellular secretory vesicles (black arrows) are present. (Proximal convoluted tubule, PCT; scale bar:500 nm, x14000).

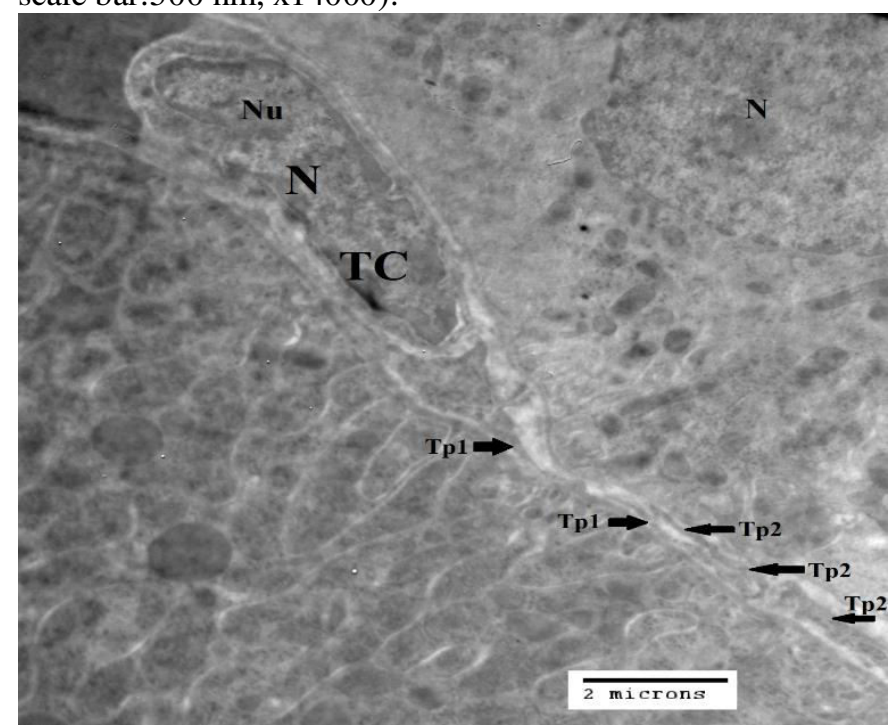

Fig.12: TEM photomicrograph of ultrathin section from the kidney in the group II. Telocyte (TC) is present in between the renal tubules. TC display a relatively large indented oval nucleus $(\mathrm{N})$ showing patches of heterochromatin particularly near the nuclear membrane in addition to euchromatin, nucleolus $(\mathrm{Nu})$ is apparant. The nucleus is surrounded by a thin cytoplasmic layer containing cytoplasmic organelles. Telopodes (Tp1) arise from the TC. Telopode (Tp2) of another TC is present. (Scale bar: $2 \mathrm{um}, \mathrm{x} 7200$ ).

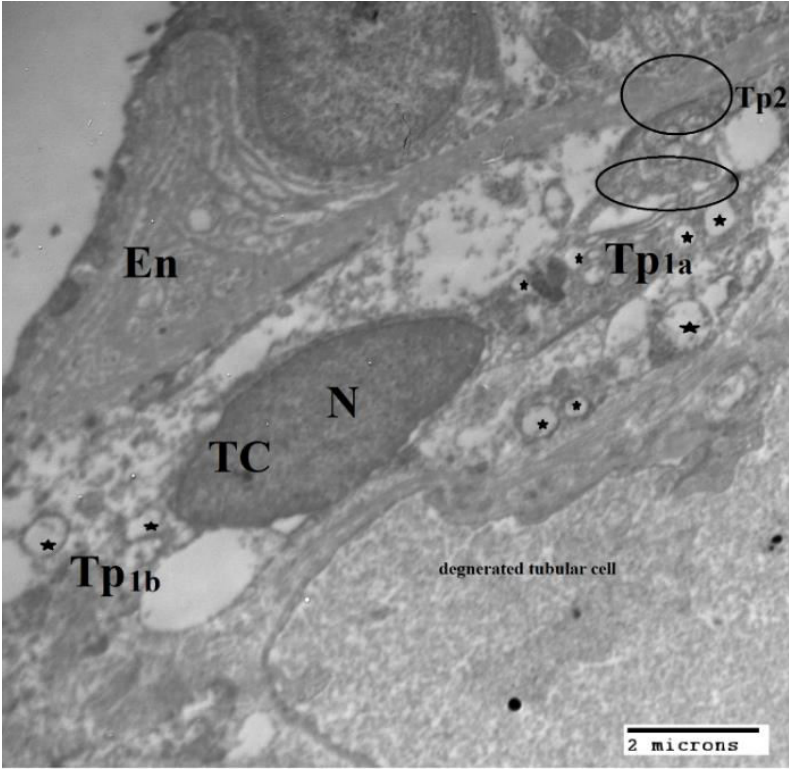

Fig.13: Transmission electron photomicrograph of ultrathin section from the kidney in the group III. Telocyte (TC) is present in renal interstitium with the telopode (Tp1a and Tp1b) extending from it. The nucleus (N) of the TC a is relatively large and shows condensed chromatin. The perinuclear cytoplasm is reduced with marked vacuolization of the cytoplasm in Tp. Many intra- and extra-cytoplasmic vesicles are present (asterisks). Parts of the cellular membrane are dissoluted with extrusion of degenerated cytoplasmic contents. Homo- and hetero-cellular junctions (circles) are present between TP2 with the endothelial cell (En) and with Tp1a of the adjacent TC respectively. Degenerated tubular cell is present. (Scale bar: 2 um, x7200).

Table (1): The mean values \pm standard deviation $($ mean \pm SD) of the morphometric measurements of TCs in the groups I, II, and III:

\begin{tabular}{|c|c|c|c|}
\hline parameters & $\begin{array}{l}\text { Group I } \\
\text { (3 weeks) }\end{array}$ & $\begin{array}{l}\text { Group II } \\
\text { (3 month) }\end{array}$ & $\begin{array}{l}\text { Group III } \\
\text { (1 year) }\end{array}$ \\
\hline $\begin{array}{l}\text { Number of } \\
\text { TCs/ hpf } \\
\text { P value }\end{array}$ & $5.05 \pm 1.20$ & $\begin{array}{l}3.0 \pm 0.93 \\
{ }^{1} \mathrm{P}<0.0001^{* * *}\end{array}$ & $\begin{array}{l}1.83 \pm 0.81 \\
{ }^{1} \mathrm{P}<0.0001^{* * *} \\
{ }^{2} \mathrm{P}<0.0001^{* * *}\end{array}$ \\
\hline $\begin{array}{l}\text { TCs cell body } \\
\text { length (um) } \\
P \text { value }\end{array}$ & $8.49 \pm 2.14$ & $\begin{array}{l}6.66 \pm 1.60 \\
0.0478^{* 1}\end{array}$ & $\begin{array}{l}5.43 \pm 2.69 \\
0.0181^{* 1} \\
0.1584(\mathrm{~ns})^{2}\end{array}$ \\
\hline $\begin{array}{l}\text { TCs nucleus } \\
\text { diameter (um) } \\
\text { P value }\end{array}$ & $1.55 \pm 0.19$ & $\begin{array}{l}1.35 \pm 0.46 \\
0.1521(\mathrm{~ns})^{1}\end{array}$ & $\begin{array}{l}2.03 \pm 0.59 \\
0.0333^{* 1} \\
0.0170^{* 2} \\
\end{array}$ \\
\hline $\begin{array}{l}\text { Length of Tps } \\
\text { (um) } \\
\text { P value }\end{array}$ & $10.79 \pm 4.39$ & $\begin{array}{l}9.61 \pm 3.53 \\
0.2804(n s)^{1}\end{array}$ & $\begin{array}{l}7.96 \pm 3.37 \\
0.0693(\mathrm{~ns})^{1} \\
0.1646(\mathrm{~ns})^{2} \\
\end{array}$ \\
\hline Number & $2.57 \pm 0.79$ & $2.0 \pm 0.58$ & $1.71 \pm 0.49$ \\
\hline
\end{tabular}




\begin{tabular}{|l|l|l|l|}
\hline Tps & & $0.0736(\mathrm{~ns})^{1}$ & $0.0153^{* 1}$ \\
P value & & $0.1685(\mathrm{~ns})^{2}$ \\
\hline
\end{tabular}

Values are mean $\pm S D,{ }^{1}$; $p$ value in comparison with group I, ${ }^{2}$; $\mathrm{p}$ value in comparison with group II ns: non-significant $(\mathrm{P}>0.05)$,

$*$ P-value $<0.05, * *$ P-value $<0.01 ; * * *$ P-value $<0.001$

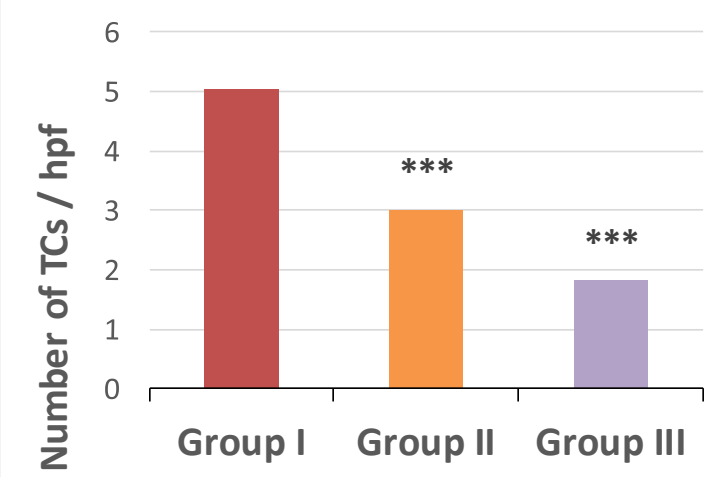

Chart (1): Showing the column graphic presentation for the mean values of number of TCs in groups I, II, and III (***)statistical significant difference in comparison to group I).

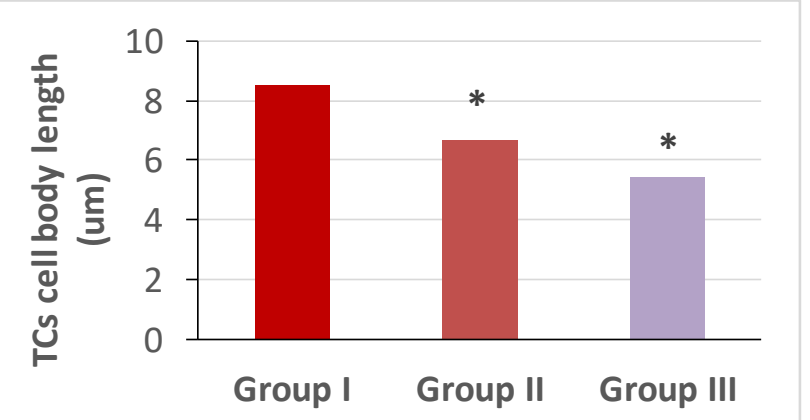

Chart (2): Showing the column graphic presentation for the mean values of TCs cell body length (um) in groups I, II, and III (*statistical significant difference in comparison to group I).

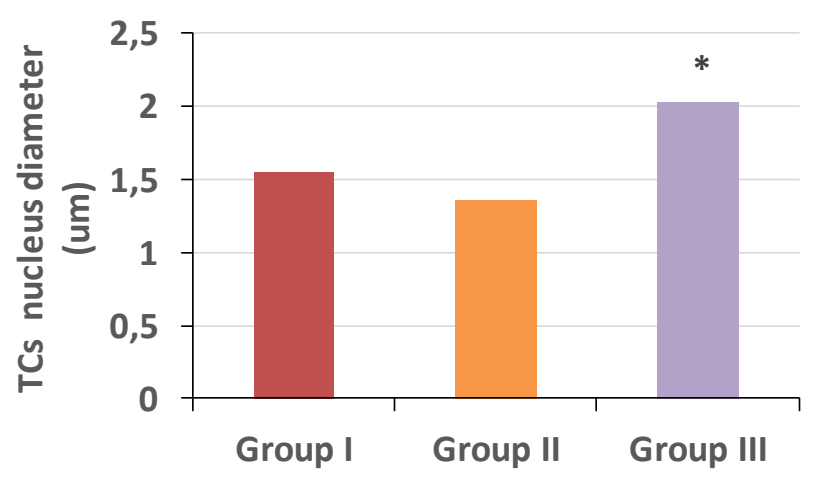

Chart (3): Showing the column graphic presentation for the mean values of TCs nucleus diameter (um) in groups I, II, and III.

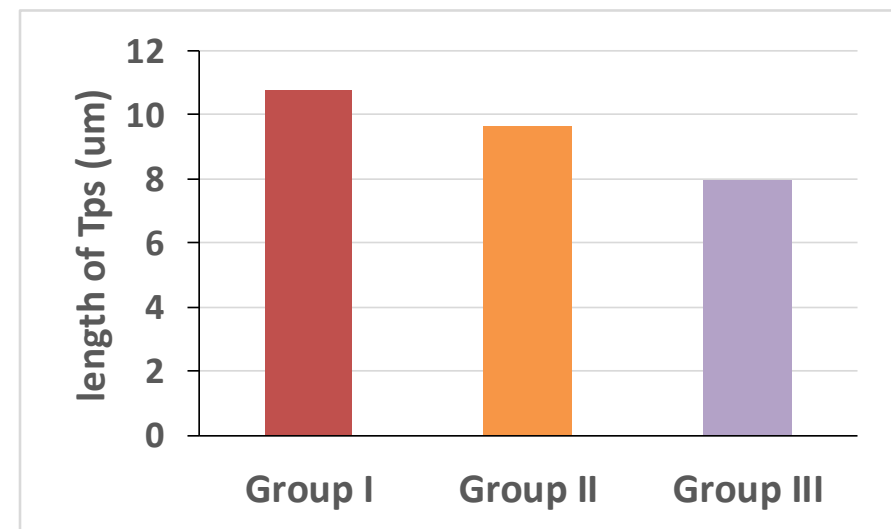

Chart (4): Showing the column graphic presentation for the mean values of length of Tps (um) in groups I, II, and III.

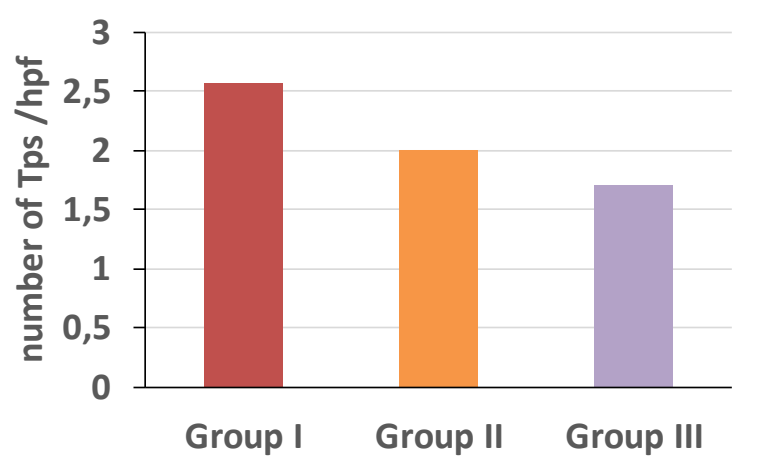

Chart (5): Showing the column graphic presentation for the mean values of number of Tps/ hpfin groups I, II, and III.

\section{Discussion:}

Since telocytes (TCs) have been identified by Popescu and Faussone-Pellegrini (2010), many researches on TCs have proved its existence, structure and specific immune markers expression in many normal organs and in different species. Multiple physiologic functions have been suggested for these cells. Bei et al. (2016) and Gandahi et al. (2020) reported that TCs have been implicated in angiogenesis, cellular signaling, tissue homoeostasis, cell expansion and movement, remodeling and repair, and they suggested to suppress oxidative stress and cellular ageing, and may have a protective effect against inflammation and oncogenesis.

Kidney aging is a normal physiological process associates with various morphologic, molecular and functional changes in the kidney 
tissues. In the present study, renal tubular changes are observed in H\&E stained sections of renal cortical tissue in group III (12 months, late adult group) in the form of vacuolation in the cells of renal tubules, degenerated cells, and congestion of blood vessels, although these changes are not noticeable in either group I (3-week aged) or group II (3-month aged group). This result is in agreement with the findings of Zhou et al. (2008)and Kotob et al. (2021) in their studies on kidney aging as regard the tubulointerstitial findings.

Miyazawa et al. (2009), Small et al. (2012) and O'Sullivan et al. (2017) suggested in their studies on the aged rat kidneys that the increased oxidative load (including mitochondrial oxidative phosphorylation) in the aging kidney together with decreased antioxidative capacity, reduced levels of $\mathrm{Cu} / \mathrm{Zn}-\mathrm{SOD}$ catalase, and GSH reductase may be responsible for chronic cellular stress, mitochondrial injury, apoptosis and cellular damage.

Morphometric study and analysis of TCs in the kidney are accomplished in our study through counting TCs in CD34-immunostained sections. This present study shows that the number of TCs decrease with the advancement of age, where there is a significant decrease (P-value $<0.0001)$ in number of TCs in each of group II and III in comparison with those in group I. Also there is a significant decrease (P-value $<0.0001$ ) in number of TCs in group III in comparison with group II. This result is consistent with the results found by Popescu et al. (2015) in their study on the cardiac TCs where they found that the number of TCs is significantly decreased (P-value $<0.01)$ in children and adults compared with the newborn group, they suggested that TCs are the first cells involved in the heart regeneration and their reduction with aging is associated with limited regenerative or reparative ability of myocardium with the advancement of age.

Examination of semithin sections of specimens of group I and II stained with toluidine blue shows TCs with their characteristic Tps in between the renal tubules and in close proximity to the blood vessels and glomeruli. Whereas in group
III, TCs are identified, but they are few and far between, which is in agreement with our results of the significant reduction of the TCs number in group III in comparison to those in groups I and II as detected in the CD34-immunostained sections.

The ultrastructural findings of TCs in the renal specimens in the groups I and II are in agreement with those documented by Gherghiceanu and Popescu (2011) and Zheng et al. (2012). TEM examination in group III renal specimens shows that TCs are rare as they are found with difficulty in the renal cortical tissue, in addition to that disintegrated TCs with fragmentation of their Tps are observed, a result that is not observed in either group I or II ultrathin sections. This result is consistent with Zhou et al. (2008), Melchioretto et al. (2016) and Kotob et al. (2021), who found in their studies that renal aging involves several tubulointerstitial degenerative changes in kidney structure in the form of tubulointerstitial fibrosis, apoptosis and atrophy with inflammatory cell interstitial infiltration and these alterations interfere with the physiologic functions and end with chronic renal failure. They explained their results by the presence of arterial intimal fibrosis demonstrated in the aged kidney groups with the development of renal ischemia and other glomerular and tubulointerstitial degenerative changes. So that the reduction of TCs count with aging in addition to the TCs disintegration in our study could be a part of generalized reduction in the renal mass and the degenerative tubulointerstitial changes with aging.

In our study the significant reduction of TCs count with advancement of age (groups II and III) in comparison to the early age group (group I) may be associated with the reduced ability of the kidney to regenerate following the age-related degenerative changes with apoptosis in advancing age groups. This is in agreement with Li et al. (2014) who in their study suggested that the presence of TCs in the kidney have a potential role in the repair and regeneration of injured tissues. Where TCs transplantation could effectively reduce the renal histological damage and attenuate renal dysfunction 
after renal ischaemia-reperfusion injury (IRI) in rats.

In the present study there is a significant decrease $(\mathrm{P}$-value $<0.05)$ in the mean value of the length of TCs' cell body in the groups II and III in comparison with the mean value in group I TCs. While there is non-significant decrease of the mean value of the length of TCs cell body (P-value > 0.05 ) in group III in comparison to group II mean value. This is in agreement with Tamura et al. (2021) who reported that TCs undergo cellular atrophy and shrinkage as a normal change with aging, with reduced immunoreactivity and expression of genes in the TCs in the aged rat stomach specimens.

There is a non-significant decrease $(\mathrm{P}$-value $>0.05)$ of the mean value of the diameter of TCs nuclei in group II rats in comparison with those in group I. Also, there is a significant increase ( $\mathrm{P}$ value $<0.05)$ in the mean value of the diameter of TCs nuclei in group III rats in comparison with those in each of group I and in group II TCs. This finding is in consistence with the changes in the mean value of the diameter of cell body in the TCs in the groups II and III. This is supported by the study of Webster et al. (2009) who reported that the nuclear and cytoplasmic volumes are somehow related to each other with a constant nucleocytopasmic ratio throughout the cell cycle.

Through the TEM examination of TCs, Tps are detected as discontinuous segments emerging from the TCs' cell body with alternation of podoms and podomers. Tps are shown to contain cytoplasm with typical cellular organelles, especially mitochondria and vesicles. Tps were described by Zhong et al. (2018)as being specific and characteristic for TCs, as Tps existed forming a 3dimensional network associated with the blood vessels, capillaries, smooth muscles and never endings.

Secretory vesicles and caveolaecould be abundantly detected on ultrastructural examination in Tps of groups I and III than in group II. Their abundance at the early age group (group I) could be supported by the result of the study by Hussein and
Mokhtar (2018) on the TCs throughout lung development, who detected an increase in the number of secretory vesicles and caveolae in postnatal life and suggested that they are concerned with secretory activity and consequent transport of signaling molecules and important macromolecules to neighboring cells. These properties enable the TCs to affect or control the activity of the surrounding cells. On the other hand, the increase of the secretory vesicles and caveolae detected in the present study in TCs and their Tps of group III is in accordance with Popescu et al. (2015) study on cardiac TCs. Where they found that the total area of mitochondria is decreased and the number of secretory vesicles and caveolae is higher in the aged rat group in comparison to the newly born group. They attributed this result to the possible contributive role of $\mathrm{TCs}$ in the repair and regeneration of the surrounding tissue during aging through massive release of secretory vesicles containing macromolecular signals as microRNAs to the extracellular matrix.

There is a non-significant decrease (P-value $>0.05$ ) of the mean value of the Tps length of TCs in renal cortical tissue in group II rats in comparison with those in group I. Also, there is a nonsignificant decrease (P-value > 0.05) in the mean value of the Tps length in group III rats in comparison with those in each of group I and in group II TCs. Our explanation for this age-related non-significant reduction in the Tps' length is being a part of the age-related degenerative changes in the advancing age groups with fragmentation of Tps as is clearly seen by TEM in group III ultrathin renal cortical tissue specimens.

There is a non-significant decrease (P-value $>0.05)$ of the mean value of the number of Tps emerging from each cell of TCs in renal cortical tissue in group II rats in comparison with those in group I. There is a significant decrease (P-value < 0.05 ) in the mean value of the number of Tps in group III rats in comparison with those in group I. This result is inconsistent with Enciu and Popescu (2015) who through their study on the in vitro culturing of TCs to passage 21 , documented that 
ageing of TCs is associated with an increase of the ratio of Tps/TC number with about $50 \%$.

On TEM examination of renal TCs in the groups I, II and III, homo- and hetero-cellular junctions are noticed between TCs' cell bodies with their Tps and the neighboring TCs, the cells of renal tubules and vascular endothelial cells. FaussonePellegrini and Gherghiceanu (2016) and Cretoiu et al. (2017) documented that the TCs by their extremely long and interconnecting Tps, form a 3dimensional labyrinthine network and by means of their homocellular contacts could function as a scaffold necessary to determine the correct parenchymal organization during morphogenesis or to drive tissue renewal or repair in the postnatal life. In addition to the homocellular contacts, TCs establish heterocellular contacts with most of cell types. Through both types of contact, TCs are involved in the intercellular exchange and electrical signaling to maintain tissue/organ function.

\section{Conclusion:}

The present study provided an evidence of the presence of TCs in the interstitium of the renal cortex as evidenced by light and electron microscopy. On comparison of the age groups in the study, it is proved that TCs decrease in number with degenerative changes observed with aging. This could be associated with decreased regenerative capacity of the renal tissue with advancement of age.

\section{References:}

Ayache J, Beaunier L, Boumendil J, Ehret G, Laub D (2010). Sample Preparation Handbook for Transission Electron Microscopy.

Bancroft JD, Layton C (2013). Theory and Practice of Histological Techniques. 7th Edition.

Bei Y, Zhou Q, Sun Q, Xiao J (2016). Telocytes in cardiac regeneration and repair. Seminars in cell \& developmental biology, 55:14-21.

Bussolati B, Hauser PV, Carvalhosa R, Camussi G (2009). Contribution of stem cells to kidney repair. Current stem cell research \& therapy, 4:2-8.
Cismasiu VB, Popescu LM (2015). Telocytes transfer extracellular vesicles loaded with microRNAs to stem cells. Journal of cellular and molecular medicine, 19:351-358.

Cretoiu D, Radu BM, Banciu A, Banciu DD, Cretoiu SM (2017). Telocytes heterogeneity: From cellular morphology to functional evidence. Seminars in cell \& developmental biology, 64:26-39.

Cretoiu D, Xu J, Xiao J, Cretoiu SM (2016). Telocytes and Their Extracellular VesiclesEvidence and Hypotheses. International journal of molecular sciences, 17.

Enciu AM, Popescu LM (2015). Telopodes of telocytes are influenced in vitro by redox conditions and ageing. Molecular and cellular biochemistry, 410:165-174.

Faussone-Pellegrini MS, Gherghiceanu M (2016). Telocyte's contacts. Seminars in cell \& developmental biology, 55:3-8.

Faussone-Pellegrini MS, Popescu LM (2011). Telocytes. Biomolecular concepts, 2:481489.

Gandahi NS, Ding B, Shi Y, Bai X, Gandahi JA, Vistro WA, et al. (2020). Identification of Telocytes in the Pancreas of Turtles-A role in Cellular Communication. International journal of molecular sciences 21 .

Gherghiceanu M, Popescu LM. (2011). Heterocellular communication in the heart: electron tomography of telocyte-myocyte junctions. Journal of cellular and molecular medicine, 15:1005-1011.

Hussein MM, Mokhtar DM. (2018). The roles of telocytes in lung development and angiogenesis: An immunohistochemical, ultrastructural, scanning electron microscopy and morphometrical study. Developmental biology 443:137-152.

Kotob MH, Hussein AM, Abd-Elkareem M. (2021). Histopathological changes of kidney tissue during aging. SVU- International Journal of Veterinary Sciences, 4:54-65.

Li L, Lin M, Li L, Wang R, Zhang C, Qi G, et al. (2014). Renal telocytes contribute to the repair of ischemically injured renal tubules. 
Journal of cellular and molecular medicine, 18:1144-1156.

Liao Z, Chen Y, Duan C, Zhu K, Huang R, Zhao H, et al. (2021). Cardiac telocytes inhibit cardiac microvascular endothelial cell apoptosis through exosomal miRNA-21-5ptargeted cdip1 silencing to improve angiogenesis following myocardial infarction. Theranostics, 11:268-291.

Marini M, Mencucci R, Rosa I, Favuzza E, Guasti D, Ibba-Manneschi L, et al. (2017). Telocytes in normal and keratoconic human cornea: an immunohistochemical and transmission electron microscopy study. Journal of cellular and molecular medicine, 21:3602-3611.

Melchioretto EF, Zeni M, Veronez DA, Martins ELF, Fraga R. (2016). Quantitative analysis of the renal aging in rats. Stereological study. Acta cirurgica brasileira, 31:346-352.

Miyazawa M, Ishii T, Yasuda $\mathbf{K}$, Noda S, Onouchi H, Hartman PS, et al. (2009). The role of mitochondrial superoxide anion $(\mathrm{O} 2(-))$ on physiological aging in C57BL/6 J mice. Journal of radiation research, 50:7383.

O'Sullivan ED, Hughes J, Ferenbach DA. 2017. Renal Aging: Causes and Consequences. Journal of the American Society of Nephrology : JASN 28:407-420.

Popescu LM, Curici A, Wang E, Zhang H, Hu S, Gherghiceanu M. (2015). Telocytes and putative stem cells in ageing human heart. Journal of cellular and molecular medicine, 19:31-45.

Popescu LM, Faussone-Pellegrini MS. (2010). TELOCYTES - a case of serendipity: the winding way from Interstitial Cells of Cajal (ICC), via Interstitial Cajal-Like Cells (ICLC) to TELOCYTES. Journal of cellular and molecular medicine, 14:729-740.

Small DM, Bennett NC, Roy S, Gabrielli BG, Johnson DW, Gobe GC. (2012). Oxidative stress and cell senescence combine to cause maximal renal tubular epithelial cell dysfunction and loss in an in vitro model of kidney disease. Nephron Experimental nephrology, 122:123-130.

Song B, Smink AM, Jones CV, Callaghan JM, Firth SD, Bernard CA, et al. (2012). The directed differentiation of human iPS cells into kidney podocytes. PloS one, 7:e46453.

Tamura Y, Takata K, Eguchi A, Maeda M, Kataoka Y. (2021). Age-related changes in NG2-expressing telocytes of rat stomach. PloS one, 16:e0249729.

Wang L, Xiao L, Zhang R, Jin H, Shi H. (2020). Ultrastructural and immunohistochemical characteristics of telocytes in human scalp tissue. Scientific reports, 10:1693.

Webster M, Witkin KL, Cohen-Fix O. (2009). Sizing up the nucleus: nuclear shape, size and nuclear-envelope assembly. Journal of cell science, 122:1477-1486.

Xu Y, Tian H, Cheng J, Liang S, Li T, Liu J. (2019). Immunohistochemical biomarkers and distribution of telocytes in ApoE(-/-) mice. Cell biology international, 43:12861295.

Zhang H, Zhong S, Ge T, Peng S, Yu P, Zhou Z, et al. (2016). Telocytes in ileum of the Chinese giant salamander: ultrastructural evidence. Journal of cellular and molecular medicine, 20:568-574.

Zheng Y, Zhu T, Lin M, Wu D, Wang X. (2012). Telocytes in the urinary system. Journal of translational medicine, 10:188.

Zhong X, Zheng Y, Li H, Huang S, Ge J. (2018). Identification of Myocardial Telocytes and Bone Marrow Mesenchymal Stem Cells in Mice. Cell transplantation, 27:1515-1522.

Zhou XJ, Rakheja D, Yu X, Saxena R, Vaziri ND, Silva FG. (2008). The aging kidney. Kidney international, 74:710-720. 